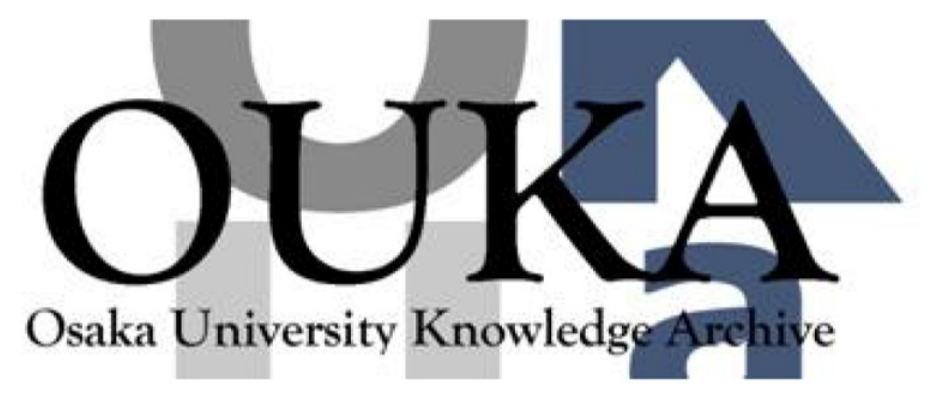

\begin{tabular}{|c|l|}
\hline Title & $\begin{array}{l}\text { Demonstration of ObserVAR: Visualization System } \\
\text { for Observing Virtual Reality Users using } \\
\text { Augmented Reality }\end{array}$ \\
\hline Author(s) & $\begin{array}{l}\text { Thanyadit, Santawat; Punpongsanon, Parinya; } \\
\text { Pong, Ting-Chuen }\end{array}$ \\
\hline Citation & SIGGRAPH Asia 2020 XR, SA 2020. p. 13 \\
\hline Issue Date & $2020-12-04$ \\
\hline oaire:version & AM \\
\hline URL & https://hdl. handle. net/11094/78270 \\
\hline rights & \\
\hline Note & \\
\hline
\end{tabular}

Osaka University Knowledge Archive : OUKA

https://ir. Library. osaka-u. ac. jp/

Osaka University 


\section{Demonstration of ObserVAR: Visualization System for Observing Virtual Reality Users using Augmented Reality}

\author{
Santawat Thanyadit \\ santawat@ust.hk \\ The Hong Kong University of Science \\ and Technology \\ Clear Water Bay, Hong Kong
}

\author{
Parinya Punpongsanon \\ parinya@sys.es.osaka-u.ac.jp \\ Osaka University \\ Toyonaka, Osaka
}

\author{
Ting-Chuen Pong \\ tcpong@ust.hk \\ The Hong Kong University of Science \\ and Technology \\ Clear Water Bay, Hong Kong
}

\begin{abstract}
Virtual Reality (VR) has been used increasingly as a part of VR classroom in which students can explore outside the classroom experiences inside the classroom. However, the instructor is unaware of the students' activities and engagement inside the virtual experiences, because the instructor cannot observe a large group of students inside the VR classroom. Thus, it hinders interactions between the instructor and students. To solve this challenge, we present a visualization method that allows the instructor to observe VR users (i.e., students) at scale using Augmented Reality. Specifically, the virtual environment and students' gazes are visualized for the instructor and optimized to reduce visual clutter, so the instructor has overall awareness of the entire VR classroom.
\end{abstract}

\section{CCS CONCEPTS}

- Human-centered computing $\rightarrow$ Interaction techniques; $\mathrm{Vi}$ sualization application domains.

\section{KEYWORDS}

interactive visualization, remote collaboration, VR classroom, asymmetric interaction

\section{ACM Reference Format:}

Santawat Thanyadit, Parinya Punpongsanon, and Ting-Chuen Pong. 2020. Demonstration of ObserVAR: Visualization System for Observing Virtual Reality Users using Augmented Reality. In SIGGRAPH Asia 2020 XR (SA '20 XR), December 01-09, 2020. ACM, New York, NY, USA, 2 pages. https: //doi.org/10.1145/3415256.3421495

\section{INTRODUCTION}

Virtual Reality (VR) has been used increasingly as a part of VR classroom in which students can explore outside the classroom experiences inside the classroom. In a traditional method, an instructor has to use a computer monitor to spectate students one-by-one [4], however, such method is insufficient for a class of students. Furthermore, the instructor can only see the virtual environment (VE) through the students' view via the monitor, which is difficult for the instructor to gain an overall awareness of the VR classroom and provide detailed instructions.

Permission to make digital or hard copies of part or all of this work for personal or classroom use is granted without fee provided that copies are not made or distributed for profit or commercial advantage and that copies bear this notice and the full citation on the first page. Copyrights for third-party components of this work must be honored.

For all other uses, contact the owner/author(s).

SA '20 XR, December 01-09, 2020, Virtual Event, Republic of Korea

(C) 2020 Copyright held by the owner/author(s).

ACM ISBN 978-1-4503-8111-6/20/11.

https://doi.org/10.1145/3415256.3421495
Existing Approach

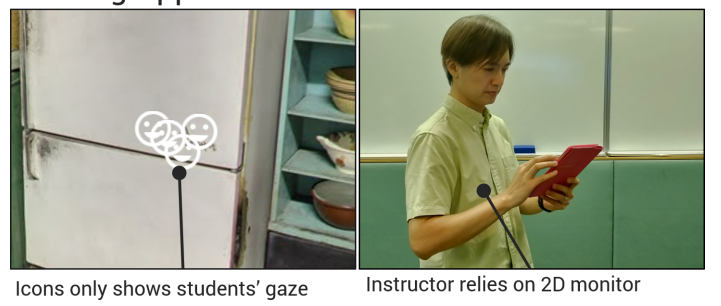

Our Approach

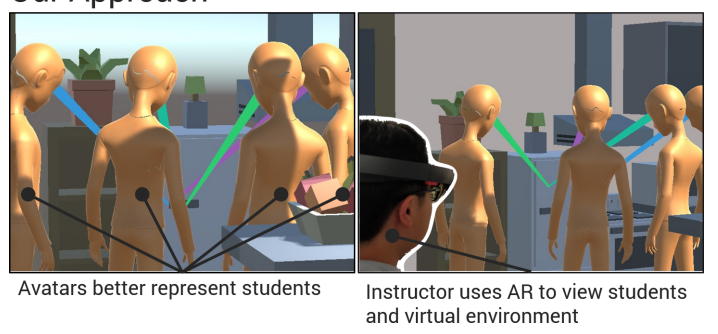

Figure 1: Differences between an existing approach and our proposed method. In existing approach, instructor uses an icon to represent each student, which is relies on the $2 \mathrm{D}$ monitor. In our approach, the avatars represents the students. So that instructor uses augmented reality (AR) to observe and instruct each student in VR classroom.

Previous work has been investigated the method that allows the remote supervisor to observe and instruct a local worker by visualizing the head pose and visual cue using Augmented Reality (AR). The system could helps the local workers to understand where they should interact with the objects in the scene [1]. In an educational situation, recent VR products such as Google Expedition allow the instructor to see the virtual environment and icons which represent each student [3]. However, it is difficult for the instructor to identify which icon belongs to which student. Therefore, the instructor cannot distinguish the students who are following the class and those who are lagging behind.

We propose a prototype system for visualizing both the student's gaze direction and the virtual environment with the aim to improve the instructor's understanding of the VR class. Based on our previous studies [5], we develop an AR visualization system using a "world scale" visualization to observe and instruct a group of students together in real-time. 


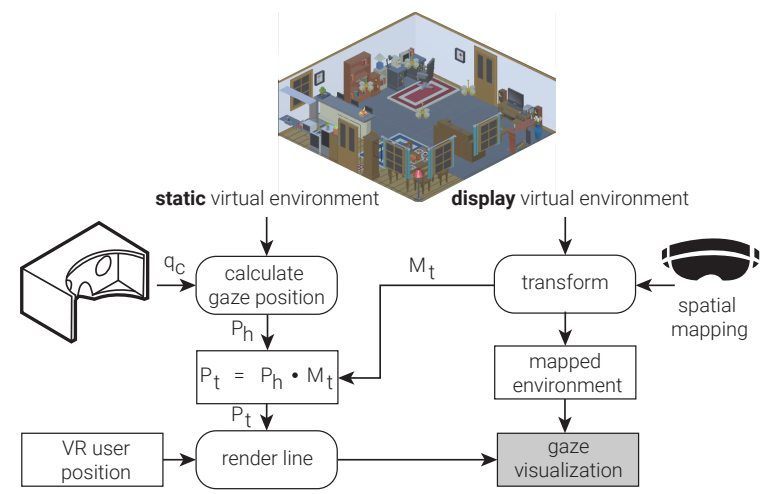

Figure 2: Transformation process to visualize the virtual environment and students' gaze directions into the instructor's physical space

\section{METHODOLOGY}

The world scale visualization considers (1) gaze and (2) environment as the important visual cue for observing and guiding students in a VR classroom. The gaze visualizes where the student is looking in the VE with an arrow which is drawn from the top of student's avatar head to a location where the student is looking at (Figure 1) The environment visualizes the VE for the instructor to understand the scene where the students are exploring. The world scale visualization maps the entire VE into the instructor's physical room using the spatial mapping tools from Microsoft HoloLens. The size of the physical room was used to change the scale of the VR and the orientation of the VE was aligned to match the physical room orientation. As shown in Figure 2, we visualize the gaze direction $q_{c}$ of the student by calculating each student's gaze position in the VE $P_{h}$. Since the VR that we used is different to the instructor's physical environment due to the spatial mapping $M_{t}$, we transform $p_{h}$ with $M_{t}$ to obtain the student's gaze position in the physical environment $P_{t}=P_{h} \cdot M_{t}$.

Finally, we reduce occlusions in the screen so the instructor gain better awareness of the VR classroom by optimizing the location of each student's avatar using a force-directed graph drawing algorithm [2].

\section{Implementation}

We implemented our prototype system using Unity3D platform with an Optical See-through HMD (Microsoft HoloLens 1) for the instructor and a VR-HMD for the students (Cardboard VR). The system runs through a PC (CPU Intel i7-7700k 4.20GHz, 16 GB of RAM with NVIDIA GPU GTX1070), which communicates with the HoloLens using a Holographic Remoting application. The VR-HMD communicate through PC via TCP/IP connections. The PC handle all the information from the VR-HMD before rendered the gaze directions of the students and VE to the HoloLens.

\section{DEMONSTRATION}

Due to the COVID-19 situation, we plan to demonstrate our work remotely by dividing our system into instructor perspective and student perspective. We plan to prepare the system where instructor

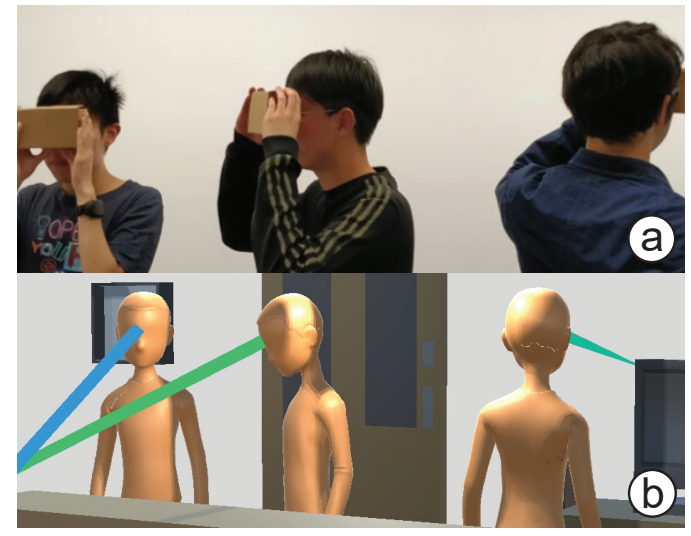

Figure 3: The visualization setting: (a) students in a remote location and (b) the visualization of students for the instructor.

perspective uses OST-HMD to visualize the students in the classroom as described in our implementation, and will be deployed locally. We will visualize the students in the VR classroom by using the pre-recorded data from the participants in our previous user study. We will stream the instructor perspective using the screen sharing during demonstration hours. If the lockdown is lifted, we also plan to broadcast two live student systems and will be shared to participants via a screen sharing software.

\section{ACKNOWLEDGMENTS}

This work was supported in part by the Hong Kong RGC's ThemeBased Research Scheme Project no. T44-707/16-N and Areas of Excellence Scheme Project no. AOE/E-603/18, the Hong Kong UGC Teaching and Learning Funding Scheme Project no. F0667, the Innovation and Technology Fund under Grant no. ITS/388/17FP, and the Osaka University Researcher Development Fund grant no. J18110201

\section{REFERENCES}

[1] Huidong Bai, Prasanth Sasikumar, Jing Yang, and Mark Billinghurst. 2020. A User Study on Mixed Reality Remote Collaboration with Eye Gaze and Hand Gesture Sharing. In Proceedings of the 2020 CHI Conference on Human Factors in Computing Systems (Honolulu, HI, USA) (CHI '20). Association for Computing Machinery, New York, NY, USA, 1-13. https://doi.org/10.1145/3313831.3376550

[2] Giuseppe Di Battista, Peter Eades, Roberto Tamassia, and Ioannis G. Tollis. 1998. Graph Drawing: Algorithms for the Visualization of Graphs (1st ed.). Prentice Hall PTR, Upper Saddle River, NJ, USA.

[3] Antigoni Parmaxi, Kostas Stylianou, and Panayiotis Zaphiris. 2018. Enabling Social Exploration Through Virtual Guidance in Google Expeditions: An Exploratory Study. In Interactive Mobile Communication Technologies and Learning. Springer International Publishing, 397-408.

[4] Santawat Thanyadit, Parinya Punpongsanon, and Ting-Chuen Pong. 2018. Efficient Information Sharing Techniques between Workers of Heterogeneous Tasks in 3D CVE. Proc. ACM Hum.-Comput. Interact. 2, CSCW, Article 172 (Nov. 2018), 19 pages. https://doi.org/10.1145/3274441

[5] Santawat Thanyadit, Parinya Punpongsanon, and Ting-Chuen Pong. 2019. ObserVAR: Visualization System for Observing Virtual Reality Users using Augmented Reality. In 2019 IEEE International Symposium on Mixed and Augmented Reality (ISMAR). 258-268. 